\title{
Bilateral Carotid Dissection Caused by a Dog Bite: Case Report and Review of the Literature
}

\author{
Limei Chen, MS ${ }^{a}$, Ananta Adhikari, MS ${ }^{a}$, Jianwen Guo, MD ${ }^{b}$, Jingxin Zhong, MS ${ }^{b}$, Guangjian Liu, MD ${ }^{a}$, \\ Feng Zhang, MS ${ }^{a \text {,* }}$ \\ ${ }^{a}$ Department of Ultrasound, Sun Yat-sen University Sixth Affiliated Hospital, Guangzhou, Guangdong Province, China; ${ }^{b}$ The \\ Cerebrovascular Disease Center, The 2nd teaching hospital of Guangzhou University of Chinese Medicine (Guangdong Provincial \\ Hospital of Traditional Chinese Medicine), Guangzhou, Guangdong Province, China
}

Received December 27, 2019; revision requested May 18, 2020; accepted May 28, 2020

\begin{abstract}
Arterial dissection is caused by intima damage and blood flow into the vascular wall, resulting in the separation of the intima and middle layer, intramural hematoma formation, and subsequently stenosis, occlusion, or aneurysm. A 68-yearold woman with bilateral common carotid artery dissection (CAD) caused by a dog bite two months prior was admitted to the hospital. Cerebral angiography, magnetic resonance angiography (MRA), and carotid color Doppler ultrasonography showed right CAD (aneurysm formation) with luminal stenosis (50\%-69\%), left common CAD (intramural hematoma formation). Bilateral common CAD caused by a dog bite is rare. The mechanism of CAD may be similar to iatrogenic dissection. After eliminating the contraindication of operation, right common carotid artery stenting was performed two months after conservative treatment.
\end{abstract}

Key words: Carotid artery dissection; Imaging; Diagnosis; Trauma

Advanced Ultrasound in Diagnosis and Therapy 2020;03:230-233

DOI: 10.37015/AUDT.2020.190042

\section{Case report}

A 68-year-old woman with bilateral common carotid artery dissection (CAD) caused by a dog bite two months prior was admitted to the hospital. The patient's symptoms included headache, weakness of both upper limbs, and no dizziness. She had a history of hypertension. A dog bite resulted in multiple open injuries of the shoulder and neck which required bilateral neck debridement and suture plus ligation of the left internal jugular vein and anastomosis of the right internal jugular vein.

On physical examination, the patient was conscious with stable vital signs, large surgical scars around the neck and medial right axilla; limited neck rotation, flexion, and extension; limited rotation of the right shoulder joint, congestion of the right inner canthus, bilateral cleft eyes, no drooping eyelids, no prominence of the eyeball, normal bilateral muscle strength, and muscle tension. Physiological but not pathological reflexes were present. Aortic arch and whole cerebral angiography showed dissection at the upper end of the right common carotid artery; distal occlusion of the right vertebral artery; and dissection formation with thrombosis at the upper part of the left common carotid artery. MRA findings of the carotid artery suggested: 1 . the possibility of dissection near the bifurcation of the right common carotid artery; and 2. right vertebral atherosclerosis with luminal stenosis. Carotid color Doppler ultrasonography showed right common CAD (aneurysm formation) with luminal stenosis $(50 \%-69 \%)$, left common CAD (intramural hematoma formation); bilateral carotid artery intimamedia thickening; and bilateral plaque formation. After eliminating the contraindication of operation, right common carotid artery stenting was performed

\footnotetext{
" Corresponding Author: Department of Ultrasound, Sun Yat-sen University Sixth Affiliated Hospital, 26 Erheng Road, Yuancun, Tianhe District, Guangzhou, Guangdong Province, China.

e-mail: sumszhangfeng@163.com unrestricted use, distribution and reproduction in any medium provided that the original work is properly attributed.
} 
two months after conservative treatment. Aspirin and clopidogrel were used to treat platelet aggregation after the operation.

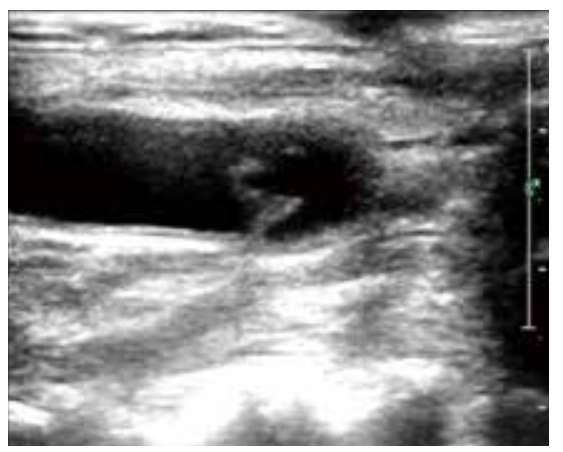

Figure 1 The longitudinal section of the right common carotid artery shows the irregular intima due to a tear.

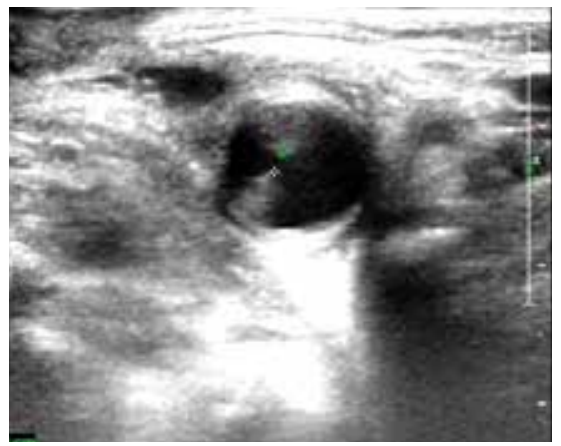

Figure 2 Cross-sectional examination of the right common carotid artery reveals a gap in the dissecting aneurysms.

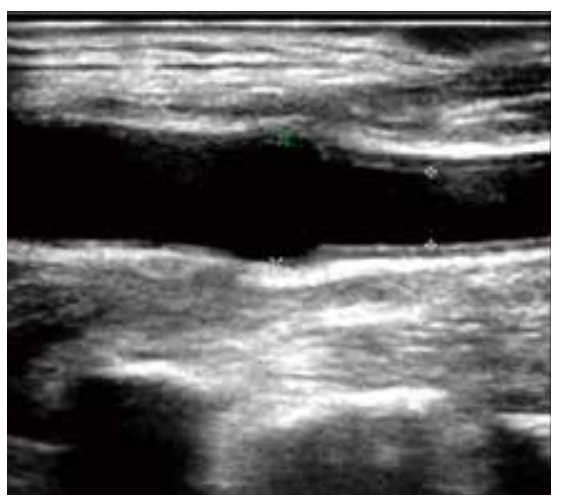

Figure 3 Localized aneurysm dilatation of the right common carotid artery.

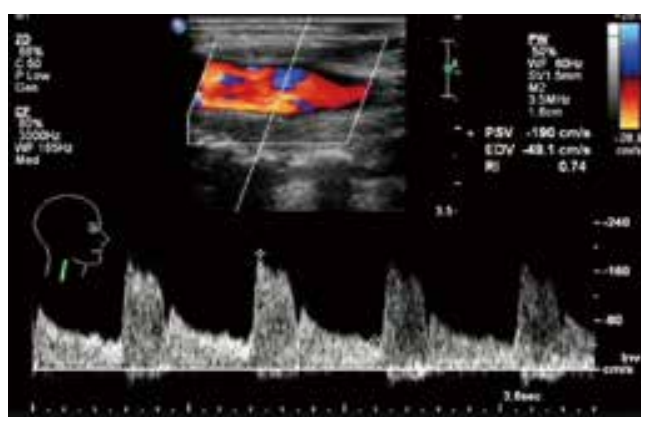

Figure 4 Stenosis of the right common carotid artery aneurysm and increased flow rate.

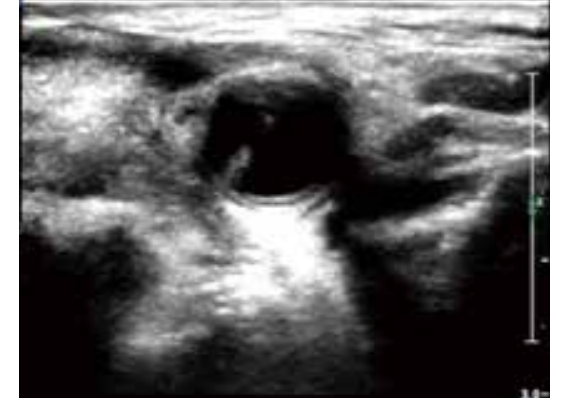

Figure 5 No significant change in the follow-up of the right common carotid artery dissecting aneurysm after two months of conservative treatment.

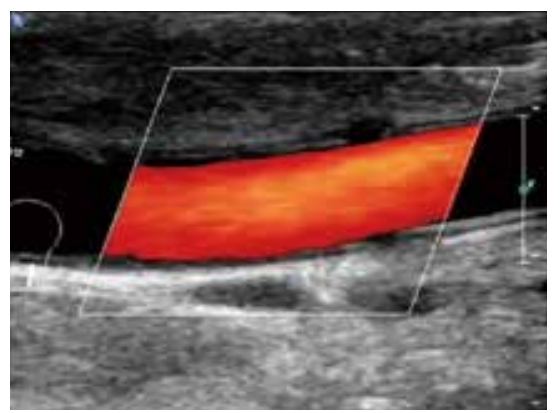

Figure 6 Intermural hematoma dissection of the anterior and posterior wall of the left common carotid artery with no perfusion of blood flow.



Figure 7 2D structure of the hematoma dissection in the anterior and posterior wall of the left common carotid artery.

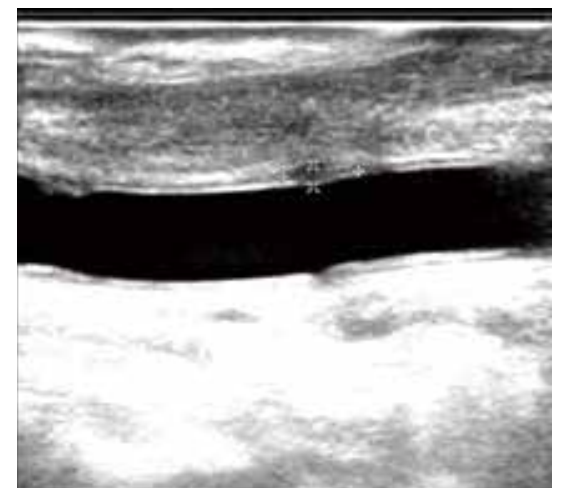

Figure 8 The left common carotid artery intramural hematoma dissection shrank after two months of conservative treatment.

\section{Discussion}

Arterial dissection is caused by intima damage and blood flow into the vascular wall, resulting in the 
separation of the intima and middle layer, intramural hematoma formation, and subsequently stenosis, occlusion, or aneurysm. As a type of arterial dissection, carotid dissection has an incidence of 2.6-3.0/100,000 person-years. It accounts for only $2 \%$ of the causes of ischemic stroke, but its proportion is as high as $8-25 \%$ among young people under 45 years old $[1,2]$.

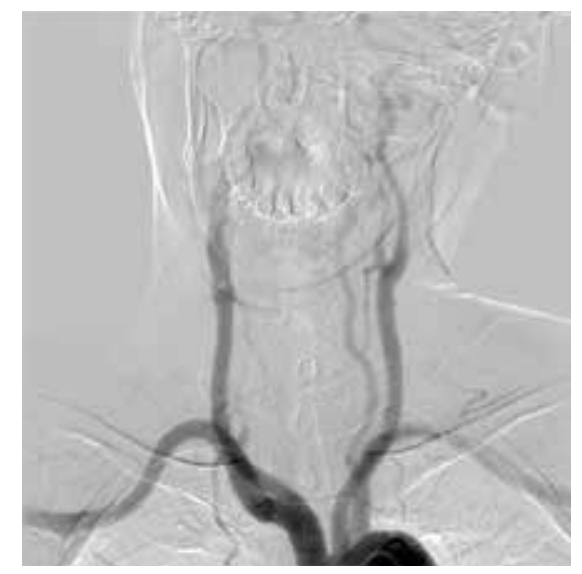

Figure 9 Positive aortic arch angiography after patient injury.

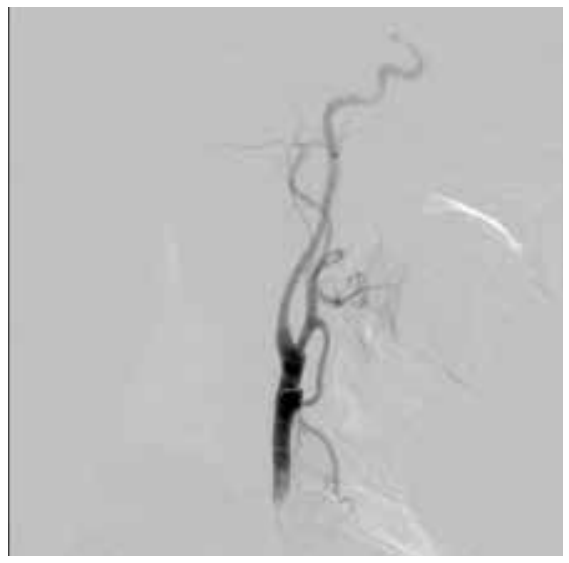

Figure 10 Angiography of the right common carotid artery following injury; uneven thickness of the right common carotid artery with dissection.

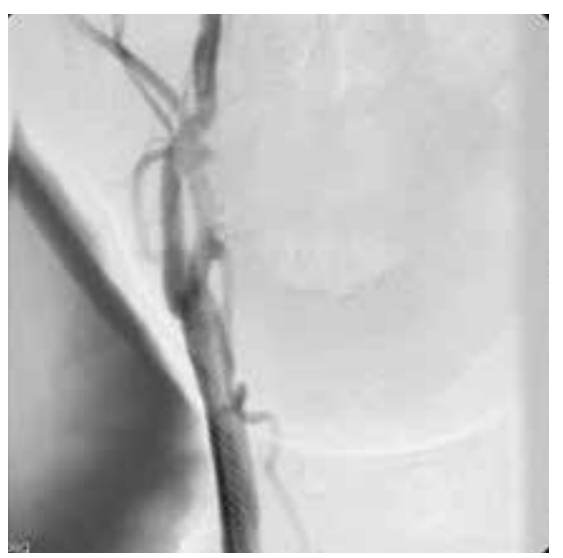

Figure 11 Right common carotid artery wallstents $(9 \times 30 \mathrm{~mm})$ after stent placement.
Most carotid dissection occurs spontaneously, and only $4 \%$ are trauma induced. Traumas are mostly nonopen, such as traffic accidents and blunt neck injuries. The causes of dissection may be excessive stretching and neck rotation leading to traction of the internal carotid artery, sudden complete flexion of the neck that directly compresses the carotid artery, or direct external force [3]. Cephalic and carotid artery dissection often occurs in large mobile vessels, and with the highest incidence in the internal carotid artery since it lacks bone structure support, followed by the vertebral and common carotid arteries [4]. Carotid dissection may occur either unilaterally or bilaterally. Common carotid dissection often occurs in aortic dissection. Primary common carotid dissection is rare. Chiba et al. [5] reviewed 17 reported cases of traumatic common CAD and found that the causes were blunt neck injuries (5 cases), traffic accidents ( 5 cases), hanging injuries (3 cases), strangulation ( 3 cases), and neck injuries ( 1 case). Only 4 of the 17 cases were bilateral common CAD, and most were related to strangulation which is directly associated with the bilateral neck.

Bilateral common CAD caused by a dog bite is rare. The mechanism of CAD may be similar to iatrogenic dissection. That is, after the dog bite, the intima-media of the carotid artery is damaged continuously after contraction and healing due to the presence of more muscle fibers in the intima-media, which results in blood flow entering the intima-media through the tear and arterial dissection formation.

The diagnosis of carotid dissection depends largely on the application of medical imaging technology. Digital subtraction angiography (DSA) can directly display arterial dissection, which has long been considered as the gold standard for CAD diagnosis. However, there are some limitations, such as its invasiveness, false negative rate (up to $17 \%$ ), inability to provide information on vascular wall changes, and inability to display secondary changes caused by CAD. Nowadays, it has been replaced by non-invasive imaging techniques, such as computed tomography angiography (CTA), magnetic resonance imaging (MRI), and ultrasound (US) [6]. Cervical color Doppler US has the advantages of being non-invasive, fast, convenient, non-radioactive, and cost-effective, among other features. Ultrasonography can directly observe the floating intima, intramural hematoma, and ruptured thrombus in the arterial cavity. It can also provide real-time blood flow velocity and resistance; and can predict the risk of vascular stenosis or occlusion, thromboembolism formation, and ischemic brain injury. However, its application is often limited by the experience of the operator. Ultrasonography is often used in clinical screening and follow-up evaluation or 
for radiography. US can also be used to examine patients with contrast agent allergies. CT/CTA and MRI/MRA have high accuracy in the diagnosis of CAD, and can also provide information on secondary changes caused by $\mathrm{CAD}$, such as cerebral infarction and subarachnoid hemorrhage. Currently, CT/CTA and MRI/MRA have replaced DSA as the main method of diagnosing CAD. When comparing the two methods, MR/MRA is less distorted by bone structure, showing the vascular structure more clearly, thus improving the sensitivity and specificity of diagnosis. Moreover, MRA does not require the injection of angiographic agents, which can reduce radiation damage and be used in patients with renal insufficiency, contrast agent allergy, and pregnancy. CT/CTA is the main technique for early diagnosis of carotid vascular lesions. CT can show high-density hematoma in the acute phase of arterial dissection, especially in patients with severe trauma. Because of the influence of the hemoglobin magnetic effect, the intramural hematoma shows different signals with time, and the early manifestation of low-equal signals is not enough to diagnose arterial dissection. CT/CTA can show more signs of dissection than MR/MRA, such as pseudoaneurysm and the intimal valve, so it is more often used in vertebral artery dissection. For evaluating internal CAD, both can be selected [7].

CAD can cause hemodynamic changes, thrombosis, or embolism due to interruption of blood perfusion, leading to central nervous system complications, such as cerebral neuropathy, acute ischemic stroke, and subarachnoid hemorrhage, so the treatment principle of these patients is mainly to prevent or delay the progress of central nervous system injury [8]. Anticoagulants and antiplatelet agents are often used to prevent thromboembolism and reduce the risk of stroke and death in CAD patients. Routine treatment is usually recommended for 3-6 months, but there is still no evidence for their use in large-sample randomized controlled studies. Intravascular or surgical treatment is usually limited to subarachnoid hemorrhage caused by ineffective drug therapy, anticoagulant contraindications, severe vascular stenosis, or markedly dilated or ruptured aneurysms [6]. At present, there is no randomized controlled study to evaluate the efficacy and safety of drug or endovascular therapies or surgical treatment for
CAD. Therefore, individualized treatment options are often needed in clinical practice.

\section{Conclusion}

This paper reports a case of bilateral common CAD caused by a dog bite in an elderly woman. The mechanism of common CAD formation induced by traumatic injury may be similar to iatrogenic arterial dissection. No similar case has been reported. Ultrasound can noninvasively observe the vascular wall changes in real time, and it is the first choice for follow-up for vascular wall trauma.

\section{Conflict of Interest}

We declare that we do not have any commercial or associative interest that represents a conflict of interest in connection with the work submitted.

\section{Reference}

[1] Biller J, Sacco RL, Albuquerque FC, Demaerschalk BM, Fayad P, Long PH, et al. American Heart Association Stroke Council. Cervical arterial dissections and association with cervical manipulative therapy: a statement for healthcare professionals from the american heart association/american stroke association. Stroke 2014; 45: 3155 74.

[2] Patel RR, Adam R, Maldjian C, Lincoln CM, Yuen A, Arneja A. Cervical carotid artery dissection: current review of diagnosis and treatment. Cardiol Rev 2012; 20: 145-52.

[3] Lee WW, Jensen ER. Bilateral internal carotid artery dissection due to trivial trauma. J Emerg Med 2000; 19: 35-41.

[4] Kalb R. Spontaneous dissection of the carotid and vertebral arteries. N Engl J Med 2001; 345(6): 467.

[5] Chiba F, Makino Y, Motomura A, Inokuchi G, Ishii N, Torimitsu $\mathrm{S}$, et al. Bilateral middle cerebral artery infarction associated with traumatic common carotid artery dissection: a case report and review of literature. Forensic Sci Int 2014; 236: e1-4.

[6] Chinese Medical Association Chinese Society of Neurology, Cerebrovascular Disease Group, Neurology Society of Chinese Medical Association. Guidelines for Diagnosis and treatment of carotid dissection in China 2015. Chinese Journal of Neurology 2015; 48: 644-651. [in Chinese]

[7] Vertinsky AT, Schwartz NE, Fischbein NJ, Rosenberg J, Albers GW, Zaharchuk G. Comparison of multidetector CT angiography and MR imaging of cervical artery dissection. AJNR Am J Neuroradiol 2008; 29: 1753-60.

[8] Flis CM, Jäger HR, Sidhu PS. Carotid and vertebral artery dissections: clinical aspects, imaging features and endovascular treatment. Eur Radiol 2007; 17: 820-34. 